\title{
Late onset recessive ataxia with Friedreich's disease phenotype
}

\author{
G DE MICHELE,* A FILla, * F BARBIERI,** A PERRETTI, $\dagger$ L SANTORO, $\dagger$ \\ L TROMBETTA, $\ddagger$ F SANTORELLI,* G CAMPANELLA* \\ From the Departments of Neurology* and Clinical Neurophysiology, $\dagger$ Second School of Medicine, Naples and \\ the Neurological Institut Sanatrix, $\ddagger$ Venafro, Italy
}

SUMMARY The Quebec Cooperative Study on Friedreich's ataxia required an onset before age 20 as an obligatory criterion of Friedreich's disease (FD). Harding included patients with onset before 25 years. We studied nine patients with FD phenotype but with onset ranging from 21 to 29 years (mean 24.4). Statistical analysis of the distribution and intrafamilial variation of onset age suggests that late onset Friedreich's disease (LOFD) is a distinct genetic entity or results from modifying genes in some families. Scoliosis was less common in LOFD than FD patients but otherwise the clinical picture was similar.

The diagnostic criteria for Friedreich's disease (FD) have recently been defined by the Quebec Co-operative study of Friedreich's Ataxia (QCSFA) ${ }^{1}$ and by Harding. ${ }^{2}$ They found that recessive inheritance, progressive ataxia, and lower limb areflexia are present in all cases, whereas extensor plantar response pes cavus, scoliosis and cardiomyopathy are frequent but not essential signs. Dysarthria, decreased lower limb deep sensation and weakness, obligatory signs for the QCSFA, are not considered essential for an early diagnosis by Harding. ${ }^{2}$ Moreover, the latest onset is 20 years for the QCSFA $^{1}$, and before 25 years for Harding. ${ }^{2}$ In the nine patients originally described by Friedreich ${ }^{3}$ onset was about the time of puberty. Most subsequent studies ${ }^{47}$ agreed that onset age is before 20 years, but Mollaret ${ }^{8}$ considered the onset possible before the age of 25 .

We observed nine patients with FD phenotype, whose onset age was after 20 . We investigated their genetic, clinical and laboratory findings and compared them with those of a group of FD patients to establish whether they represent a different genetic entity.

\section{Patients and methods}

Of the 200 patients affected by spino-cerebellar degeneration examined from 1973-87 at our Department, FD was diagnosed in 70 . The diagnostic criteria were: no evidence of

Correspondence to: Dr G De Michele, Clinica Neurologica, Seconda Facoltà di Medicina, via Pansini 5, 80131 Naples, Italy.

Received 17 November 1988.

Accepted 10 May 1989 dominant inheritance, onset by the age of 20 , progressive unremitting ataxia of limbs and gait, and absence of knee and ankle jerks. Furthermore, either dysarthria or extensor plantar responses or echocardiographic evidence of hypertrophic cardiomyopathy was present.

Another nine patients fulfilled these criteria except for age of onset, which was later; these were shown as LOFD. A thorough neurological examination was performed. Staging of the disease was evaluated according to the Inherited Ataxias Progression Scale (IAPS) ${ }^{9}$ : stage 1, asymptomatic affected sibling; stage 2, symptoms present, but mild; stage 3, patient needs constant care and is unable to work; stage 4, patient confined to wheelchair. The patients had 12-lead electrocardiogram and 2D and M-mode echocardiography. ${ }^{10}$ Electromyography was performed in the brachial biceps, abductor digiti minimi and anterior tibial muscles. Maximal motor and sensory nerve conduction (measured orthodromically) ${ }^{1112}$ were investigated in the median and tibial nerves. Visual evoked potentials (VEP) performed by means of a black and white checker-board reversal pattern and brain auditory evoked potentials (BAEP) were tested. ${ }^{13}$ The sural nerve was removed at the ankle for light microscopy, morphometric measurements and the study of teased fibres. CT was performed with particular attention to the posterior fossa.

Statistical analysis. Departure from normality of the frequency distribution of the onset age was tested by chi square. ${ }^{14}$ The segregation ratio was calculated assuming multiple incomplete ascertainment (Weinberg's proband method). ${ }^{15}$ Genetic heterogeneity was tested by the KruskalWallis test and the Pearson correlation coefficient of onset age between siblings. ${ }^{15}$

\section{Results}

General and genetic features

Three of the LOFD patients were male and six female. 


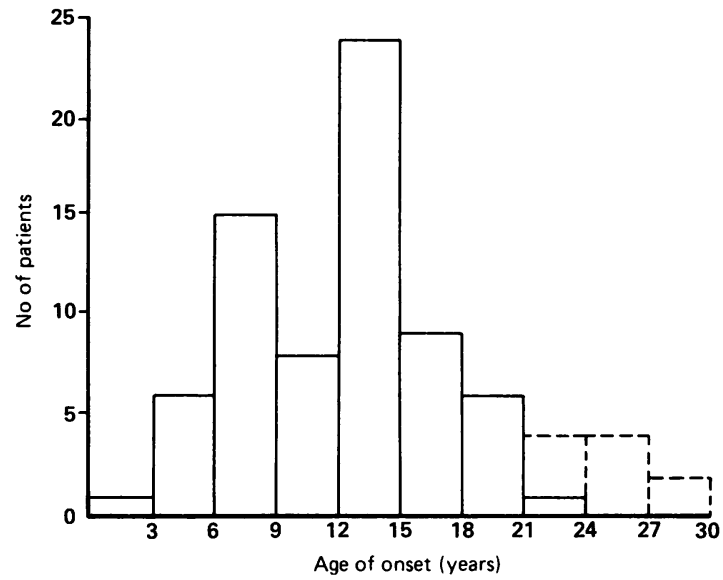

Fig Histogram of frequency distribution of onset age in 70 patients with Friedreich's disease (FD; continuous line) and nine patients with late onset Friedriech's disease (LOFD; dotted line).

Eight were index cases and one was a secondary case. The mean age (SD, range) at the time of the study was $33 \cdot 7(7 \cdot 7,23-50)$ and the mean duration of the disorder was $9 \cdot 2(7 \cdot 9,2-28)$. The patients came from six families and in three of these only one sibling was affected and two in two other families. In the sixth family two siblings were affected and one, who was dead at the time of our study, had also been affected.

In one family the parents were first cousins. The segregation ratio was 0.27 and its $95 \%$ confidence limits were $0.06-0 \cdot 49$.

The mean onset age was $24 \cdot 4(2 \cdot 7,21-29)$. The frequency distribution of age of onset of the $70 \mathrm{FD}$

Table Percent occurrence of clinical and laboratory findings in LOFD and in IAPS stage matched Friedreich's disease (FD) patients

\begin{tabular}{lcc}
\hline & $\begin{array}{c}\text { LOFD } \\
(n=9)\end{array}$ & $\begin{array}{c}F D \\
(n=27)\end{array}$ \\
\hline Gait and stance ataxia & 100 & 100 \\
Dysarthria & 100 & 78 \\
Nystagmus & 44 & 26 \\
Finger-to-nose dysmetria & 89 & 96 \\
Lower limb areflexia & 100 & 100 \\
Lower limb weakness & 56 & 63 \\
Lower limb wasting & 22 & 44 \\
Lower limb decreased vibration sense & 67 & 81 \\
Extensor plantar response & 33 & 56 \\
Pes cavus & 56 & 85 \\
Scoliosis & 33 & $93^{*}$ \\
Diabetes & 0 & 0 \\
Impaired glucose tolerance & 0 & 21 \\
Abnormal ECG & 78 & 74 \\
Abnormal echocardiogram & 22 & 33 \\
Abnormal CT-scan & 0 & 21 \\
\hline
\end{tabular}

FD patients were 21 in IAPS Stage 2, 3 in Stage 3, and 3 in Stage 4. ${ }^{*}$ Chi-square $=10.5 ; \mathrm{p}<0.01$. patients and the eight LOFD patients is shown in the figure. We did not find a significant difference from the normal distribution with the patients with FD $\left(\chi^{2}=10 \cdot 6 ; \mathrm{df}=5\right)$. On the contrary, we found a significant difference when we considered FD and LOFD patients $\left(\chi^{2}=17.9 ; \mathrm{df}=7 ; \mathrm{p}<0.05\right)$.

To investigate the possibility of genetic heterogeneity between the families with FD and LOFD, we studied the intrafamilial variation of age of onset. We preferred a non parametric test (Kruskal-Wallis test) to analysis of the variance, since the latter assumes a normal distribution. ${ }^{15} \mathrm{~S}$ value was 25.7 ( df $=15: \mathrm{p}<$ 0.05 ). We also calculated the Pearson correlation coefficient onset age between siblings, considering all possible permutations and $r_{p}$ value was $0.70(d f=42$; $\mathrm{p}<0.001$ ). When Kruskal-Wallis test and correlation coefficient were performed excluding LOFD patients, $S$ value was $16.2(\mathrm{df}=12 ; \mathrm{NS})$ and $r_{\mathrm{p}}=0.31(\mathrm{df}=36$; NS).

\section{Clinical features}

The most frequent initial symptoms in LOFD patients were gait ataxia $(78 \%)$ and lower limb weakness $(33 \%)$. The percentage frequency of clinical findings is shown in the table. Furthermore, a slight snout reflex was present in four patients, dysphagia and urinary urgency in two, perioral and lingual fasciculation in one. Seven patients were in IAPS Stage 2, one had reached Stage 3 after 10 years of the disease, and one had reached Stage 3 after eight years and Stage 4 after 28 years.

\section{Laboratory investigations}

Glucose and other routine blood tests were normal in all cases. Electrocardiographic testing revealed repolarisation abnormalities in seven patients and left ventricular hypertrophy in one of them. Echocardiograms were abnormal in two patients and suggested left ventricular hypertrophy, concentric in one case and asymmetric in the other. EEG was abnormal in two cases showing intermittent slow activity. CT, performed on five patients, was normal.

Electromyography and conduction velocity studies were performed on seven patients. Mild to moderate neurogenic abnormalities were found in the muscles that were examined. The electrophysiological findings observed in the peripheral nerves of our patients indicated a considerable decrease of amplitude or absence of sensory potentials. The mean amplitude and SD of sensory potential at the wrist was $1.53 \mu \mathrm{V}$, $2.08(10 \%$ of controls); the mean sensory orthodromic conduction velocity was $37.9 \mathrm{~m} / \mathrm{s}, 18.1(61 \%)$ and $39.0,26.7(56 \%)$ in the third digit-wrist and wristelbow segments, respectively. Motor conduction velocities were normal or slightly slowed. VEPs performed in four patients were normal. BAEPs per- 
formed in four patients were normal in two instances and showed abnormalities of the IV-V complex in the others.

Sural nerve biopsy was performed on four patients. Loss of large myelinated fibres was present in all patients and marked in three. In all subjects at least 35 teased fibres were isolated. Internodes were uniformly shortened along the entire length of most of the fibres. Some fibres presented a considerable variation of internodal length, and a few were normal.

\section{Comparison with Friedrich's disease}

Clinical and laboratory findings of LOFD patients are compared with those of FD patients in the table. Since the frequency of some features varies significantly with the progression of the disease, ${ }^{2}$ this comparison was made between LOFD patients and 27 IAPS stage matched FD patients. Statistical differences were evaluated by the chi square corrected according to Yates.

\section{Discussion}

Recognising genetic heterogeneity is important for genetic counselling, prognosis and aetiopathogenetic study. In this study, we used a genetic and clinical approach to investigate whether FD and LOFD represent the same genetic entity.

Frequency distribution of onset age significantly differed from the normal when LOFD patients were considered together with FD (figure), whereas no significant difference was found when FD patients were considered alone. Similarly, the Kruskal-Wallis test showed that the age of onset varies significantly between families only when LOFD families are included in the analysis. These data suggest the existence of more than one population. The Pearson correlation coefficient of onset age in familial cases was 0.31 in FD patients and 0.70 in FD and LOFD patients together. Haldane ${ }^{16}$ suggested that the expected correlation of some measurable characteristics between first degree relatives will be close to 0 for a single major gene with only random environmental influences, around 0.5 for modifying genes acting on one major gene and close to 1 for two or more major genes. Using this approach, FD patients appear to belong to one genetic entity, whereas LOFD could in some families represent a genetically distinct entity or the effect of modifying genes acting on the FD gene.

We compared clinical and laboratory findings of LOFD patients with those of FD patients. The lower incidence of scoliosis in LOFD patients can be explained by the later onset, after puberty. Nevertheless, the clinical picture is not clearly distinguishable from that of FD.

The electrophysiological abnormalities observed in the peripheral nerves of our patients showed a considerably decreased amplitude of the sensory potentials and a slight slowing of sensory and motor conduction velocity. These data together with the electromyographic findings are consistent with a loss of the largest peripheral nerve fibres. These data are very similar to those found in FD. ${ }^{17}$ The morphological and morphometric data from sural nerve biopsy in LOFD are the same as in FD. However, in one patient the loss of large myelinated fibres was less pronounced than is usually found in FD.

Chamberlain et al $^{18}$ reported that mapping of mutation in FD corresponds to human chromosome $19 \mathrm{p} 22-\mathrm{CEN}$. In the 22 families she studied there was no evidence of genetic heterogeneity. Winter et al ${ }^{19}$ studying intrafamilial correlation of the age of onset, suggested that FD may be genetically heterogeneous or that there are different modifying genes operating within different families. The study also considered patients with onset after age 20 . When we combined FD and LOFD patients we reached similar conclusions. Molecular genetics will clarify whether LOFD is due to a different major gene or to the presence of modifying secondary genes.

\section{References}

1 Geoffroy G, Barbeau A, Breton A, et al. Clinical description and roentgenologic evaluation of patients with Friedreich's ataxia. Canad J Neurol Scio 1976;3:279-86.

2 Harding AE. Friedreich's ataxia: a clinical and genetic study of 90 families with an analysis of early diagnostic criteria and intrafamilial clustering of clinical features. Brain 1981;104:589-620.

3 Friedreich $\mathrm{N}$. Uber degenerative atrophie der spinalen hinterstränge. Virchows Arch Path Anat 1863;26: 391-419.

4 Bell J, Carmichael EA. On hereditary ataxia and spastic paraplegia. Treasury of human inheritance, vol IV, $\mathrm{pt}$ III. Cambridge: Cambridge University Press, 1939: 141-281.

5 Greenfield JG. The spino-cerebellar degenerations. Oxford: Blackwell, 1954.

6 Tyrer JH. Friedreich's ataxia. In: Vinken PJ, Bruyn GW, eds. Handbook of clinical neurology, vol 21. Amsterdam: North Holland Publishing Company, 1975:319-64.

7 Dyck PJ, Lambert EH. Lower motor and primary sensory neuron diseases with peroneal muscular atrophy. II. Neurologic, genetic and electrophysiologic findings in various neuronal degenerations. Arch Neurol 1968;18:619-25.

8 Mollaret P. La maladie de Friedreich: étude physioclinique. Paris: Legrand, 1929.

9 Campanella G, Filla A, De Falco FA, Mansi D, Durivage A, Barbeau A. Friedreich's ataxia in the south of Italy: a clinical and biochemical survey of 23 patients. Canad J Neurol Sci 1980;7:351-8. 
10 Giunta A, Maione S, Biagini R, Filla A, De Michele G, Campanella $G$. Non invasive assessment of systolic and diastolic function in $\mathbf{5 0}$ patients with Friedreich's ataxia. Cardiology 1988;75:321-7.

11 Buchtal F, Rosenfalck A. Evoked action potentials and conduction velocity in human sensory nerves. Brain Res 1966;3:1-122.

12 Behse F, Buchtal F. Normal sensory conduction in nerves of the leg in man. J Neurol Neurosurg Psychiatry 1971; 34:404-14.

13 Pelosi L, Fels A, Petrillo A, et al. Friedreich's ataxia: clinical involvement and evoked potentials. Acta Neurol Scand 1984;70:360-8.

14 Snedecor GW, Cochran WG. Statistical methods. Ames Iowa: The Iowa State University Press, 1967:84-5.
15 Emery AEH. Methodology in clinical genetics. Edinburgh: Churchill Livingstone, 1986:37-54.

16 Haldane JBS. The relative importance of principal and modifying genes in determining some human diseases. J Genet 1941;41:149-57.

17 Caruso G, Santoro L, Perretti A, et al. Friedreich's ataxia: electrophysiologic and histologic findings in patients and relatives. Muscle and Nerve 1987;10: 503-15.

18 Chamberlain S, Shaw J, Rowland A, et al. Mapping of mutation causing Friedreich's ataxia to human chromosome 9. Nature 1988;334:248-50.

19 Winter RM, Harding AE, Baraitser M, Bravery MB. Intrafamilial correlation in Friedreich's ataxia. Clin Gen 1981;20:419-27. 